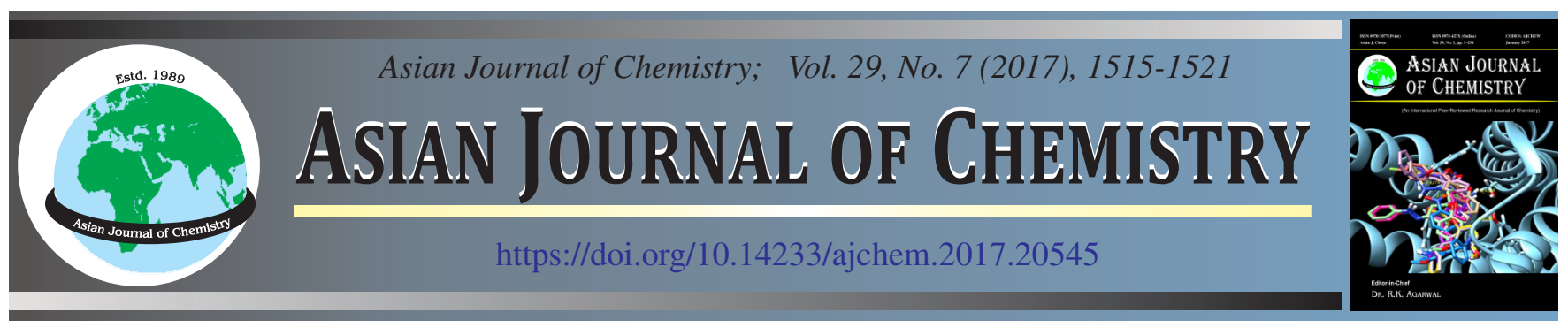

\title{
Synthesis and Antibacterial Activity of Thienopyrimidine Amide Derivatives
}

Giri Tharikoppula ${ }^{1}$, Laxminarayana Eppakayala ${ }^{1, *}$, Thirumala Chary Maringantí ${ }^{2}$, Chiranjeevi Kamalapuram ${ }^{1}$ and Karunakar Rao Kudle ${ }^{3}$

\begin{abstract}
${ }^{1}$ Sreenidhi Institute of Science and Technology, Yamnampet, Ghatkesar, Hyderabad-501301 India
${ }^{2}$ Jawaharlal Nehru Technological University Hyderabad, College of Engineering, Hyderabad-505 327, India

${ }^{3}$ Department of Chemistry, Osmania University, Main Road, Hyderabad-500 007, India
\end{abstract}

*Corresponding author: E-mail: elxnkits@yahoo.co.in

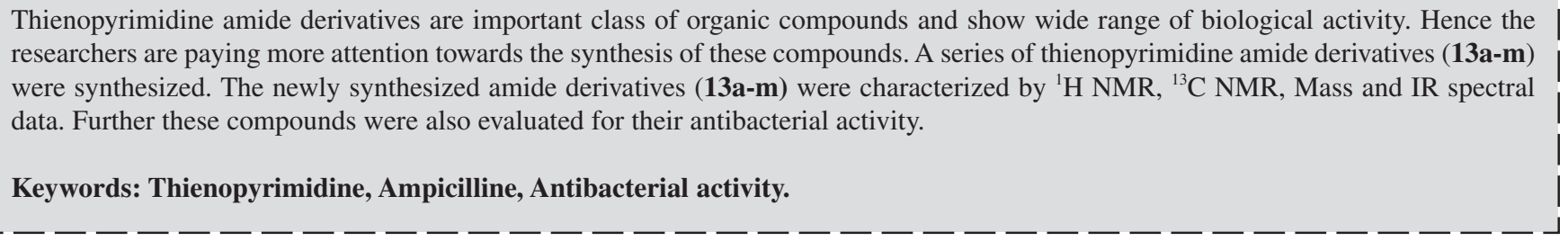

\section{INTRODUCTION}

Nearly two million cases of hospital associated infections occur annually within the U.S. The microorganism pathogens typically enter patients via canulation, urinary catheters and intravascular lines [1]. Treatments of microbic infections, that involve microorganism, is changing into tough as a result of everlasting downside of microbic resistance towards antibiotics [2]. Infections with drug resistant organisms stay a crucial downside in clinical observe that's tough to unravel. Several researchers are well productive within the recent years in reshaping the scaffolds of earlier antibiotics [3,4]. Heterocycles area unit a part of chemical science and plays a lead role within the biological activities.

Recent studies reveal that little heterocyclic frameworks, supply a plus in style of antibacterial drug agents as they mimic several biomolecules. Thienopyrimidines are nitrogen and sulfur atoms containing bicyclic heterocycle and it encompasses a thiophene ring unit with the pyrimidine moiety [5]. This scaffold shows a broad spectrum of biological activities. The thienopyrimidines were assessed as antitumour [6-9], medicine [10], enzyme [11-14], phosphodiesterase inhibitors [15], antioxidative [16,17], antimalarial drug [18,19], antibacterial drug [20], antiviral [21], antifungal [22,23], medication [24], antiplatelet [25] and medicament [26]. In view of these observations, the varied biological property of thienopyrimidine pharmacophore impelled to synthesize the title compounds. Consequently, with associate in nursing aim to develop promising antibacterial drug agents, a series of novel thienopyrimidine derivatives (13a-m) were designed and synthesized. Further, all target compounds were evaluated for antibacterial activity.

\section{EXPERIMENTAL}

The solvents were purified according to standard procedures prior to use and all commercial chemicals were as such used. For thin-layer chromatography (TLC) analysis, Merck pre-coated plates (silica gel 60 F254) were used and spots were visualized under UV light. Merck silica gel 60 (230-400 mesh) was used for flash column chromatography and the eluting solvents are indicated in the procedures. Melting point determinations were performed by using Mel-temp apparatus and were uncorrected. ${ }^{1} \mathrm{H}$ NMR and ${ }^{13} \mathrm{C}$ NMR spectra were recorded in Varian MR-400 MHz instrument. The mass spectra were recorded on Agilent ion trap MS and infrared spectra were recorded on a Perkin Elmer FT-IR spectrometer.

Synthesis of thieno[3,2-d]pyrimidine-2,4-diol (2): To a solution of methyl 3-aminothiophene-2-carboxylate (1) (10 $\mathrm{g}, 63.69 \mathrm{mmol})$ in acetic acid $(60 \mathrm{~mL})$ and water $(35 \mathrm{~mL})$ was added slowly KOCN (10.3 g, $127.3 \mathrm{mmol})$ in water $(35 \mathrm{~mL})$ by drop wise at $0{ }^{\circ} \mathrm{C}$ over a period of $30 \mathrm{~min}$ and the reaction mixture was stirred at room temperature for $16 \mathrm{~h}$. The resultant solid was filtered and treated with $50 \% \mathrm{NaOH}(40 \mathrm{~mL})$ solution and stirred at room temperature for $4 \mathrm{~h}$. After completion of the reaction, reaction mixture was cooled to $0{ }^{\circ} \mathrm{C}$ and 
acidified with conc. $\mathrm{HCl}$ provided as off white solid 2. Yield: $88 \%$; m.p: $166-170{ }^{\circ} \mathrm{C} ;{ }^{1} \mathrm{H}$ NMR (300 MHz, DMSO- $\left.d_{6}\right): \delta$ 11.55 (brs, 1H, OH), 11.20 (brs, $1 \mathrm{H}, \mathrm{OH}), 8.05$ (d, $J=5.2 \mathrm{~Hz}$, 1H, ArH), 6.91 (d, $J=5.2 \mathrm{~Hz}, 1 \mathrm{H}, \mathrm{ArH})$; ESI-MS: $m / z, 167.2$ $(\mathrm{M}-\mathrm{H})^{-}$.

Synthesis of 2,4-dichlorothieno[3,2-d]pyrimidine (3): To a suspention of thieno[3,2-d]pyrimidine-2,4-diol (2) (8.0 $\mathrm{g}, 47.61 \mathrm{mmol})$ in toluene was added $\mathrm{N}$-methyl-2-pyrrolidone (NMP) (1.0 mL, catalytic), followed by the addition and $\mathrm{POCl}_{3}$ $(35 \mathrm{~mL})$ at room temperature and heated to reflux for $16 \mathrm{~h}$. After completion of reaction, excess $\mathrm{POCl}_{3}$ was removed by distillation, crude residue was poured into ice cold water and filtered the formed precipitate compound $\mathbf{3}$ as off white solid. Yield: 7.7g, $80 \%$; m.p. 136-140 ${ }^{\circ} \mathrm{C}$; ${ }^{1} \mathrm{H}$ NMR $(300 \mathrm{MHz}$, DMSO- $\left.d_{6}\right): 8.70(\mathrm{t}, J=4.4 \mathrm{~Hz}, 1 \mathrm{H}, \mathrm{ArH}), 7.72(\mathrm{~d}, J=5.5 \mathrm{~Hz}$, 1H, ArH), ESI-MS: $m / z, 204.9(\mathrm{M}+\mathrm{H})^{+}$.

Synthesis of N-(2-chlorothieno[3,2-d]pyrimidin-4-yl)N-methylamine (5): To a solution of 2,4-dichlorothieno[3,2d]pyrimidine $(3)(7 \mathrm{~g}, 34.31 \mathrm{mmol})$ in THF $(80 \mathrm{~mL})$ was added $40 \%$ methyl amine (4) aqueous solution $(9 \mathrm{~mL})$ and stirred at room temperature for $3 \mathrm{~h}$. After completion, reaction mixture was concentrated and poured into ice water to afford solid of $\mathrm{N}$-(2-chlorothieno[3,2-d]pyrimidin-4-yl)-N-methylamine (5) as off white solid. Yield: $88 \%$; m.p.: $294-303{ }^{\circ} \mathrm{C} ;{ }^{1} \mathrm{H}$ NMR (300 MHz, DMSO- $d_{6}$ ): $\delta 8.34$ (d, $\left.J=4.7 \mathrm{~Hz}, 1 \mathrm{H}, \mathrm{ArH}\right), 8.16$ $(\mathrm{d}, J=4.7 \mathrm{~Hz}, 1 \mathrm{H}, \mathrm{ArH}), 7.32(\mathrm{~s}, 1 \mathrm{H}, \mathrm{NH}), 2.96(\mathrm{~d}, J=4.5 \mathrm{~Hz}$, 3H); ESI-MS: $m / z, 200.1(\mathrm{M}+\mathrm{H})^{+}$.

Synthesis of tert-butyl-N-(2-chlorothieno[3,2-d]pyrimidin-4-yl)-N-methylcarbamate (6): To a solution of N-(2chlorothieno[3,2-d]pyrimidin-4-yl)-N-methylamine (5) (6.0 g, $30.15 \mathrm{mmol})$ in DMF (42 mL) was added TEA (6 g, 60.30), $(\mathrm{Boc})_{2} \mathrm{O}(7.9 \mathrm{~g}, 36.18 \mathrm{mmol})$ at room temperature for $4 \mathrm{~h}$. After completion, reaction mixture was poured into ice cold water to get precipitate and dried the product under vacuum to afford pure compound 6 as off white solid. Yield: $82 \%$; m.p:126$130{ }^{\circ} \mathrm{C}$; IR $\left(\mathrm{KBr}, v_{\max }, \mathrm{cm}^{-1}\right) 3068,2978,1716,1532,1378$, $1247,1144,886,793 ;{ }^{1} \mathrm{H}$ NMR $\left(400 \mathrm{MHz}, \mathrm{DMSO}-d_{6}\right): \delta 8.51$ $(\mathrm{d}, J=5.5 \mathrm{~Hz}, 1 \mathrm{H}, \mathrm{ArH}), 7.54$ (d, $J=5.5 \mathrm{~Hz}, 1 \mathrm{H}, \mathrm{ArH}), 3.39$ (s, $\left.3 \mathrm{H}, \mathrm{CH}_{3}\right), 1.50$ (s, 9H, C( $\left.\left(\mathrm{CH}_{3}\right)_{3}\right)$; ESI-MS: $m / z, 299.9(\mathrm{M}+\mathrm{H})^{+}$.

Synthesis of tert-butyl-N-methyl-N-(2-piperazinothieno[3,2-d]pyrimidin-4-yl)carbamate (8): To a solution of piperazine (7) $(3.45 \mathrm{~g}, 40.13 \mathrm{mmol})$ in DMF $(30 \mathrm{~mL})$ at $0{ }^{\circ} \mathrm{C}$ was added potassium carbonate $(1.38 \mathrm{~g}, 10.03 \mathrm{mmol})$ followed by tert-butyl- N-(2-chlorothieno[3,2-d]pyrimidin-4-yl)-Nmethylcarbamate $(6)(3 \mathrm{~g}, 10.03 \mathrm{mmol})$ and stirred the reaction at room temperature for $12 \mathrm{~h}$. After completion, reaction mixture was poured into ice cold water, filters the formed precipitate, washed the precipitate with water and dried the product under vacuum to afford $\mathbf{8}$ as off white solid. Yield: 2.6 g. m.p. 136$140{ }^{\circ} \mathrm{C}$; IR $\left(\mathrm{KBr}, v_{\max }, \mathrm{cm}^{-1}\right): 3452,2932,2862,1711,1668$, $1567,1389,1370,1269,1151,1101,1001,763,750,662 ;{ }^{1} \mathrm{H}$ NMR (400 MHz, $\left.\mathrm{CDCl}_{3}\right): \delta 8.19-8.18(\mathrm{~d}, J=5.6 \mathrm{~Hz}, 1 \mathrm{H}$, $\mathrm{ArH}), 7.19-7.18$ (d, $J=5.6 \mathrm{~Hz}, 1 \mathrm{H}, \mathrm{ArH}), 3.80$ (brs, $4 \mathrm{H}, \mathrm{CH}_{2}$ ), $3.3\left(\mathrm{~s}, 3 \mathrm{H}, \mathrm{CH}_{3}\right), 2.93\left(\mathrm{~s}, 4 \mathrm{H}, \mathrm{CH}_{2}\right), 1.46\left(\mathrm{~s}, 9 \mathrm{H}, \mathrm{C}\left(\mathrm{CH}_{3}\right)_{3}\right)$; ESI-MS: $m / z, 359.3(\mathrm{M}+\mathrm{H})^{+}$.

Synthesis of ethyl 2-(4-4-[(tert-butoxycarbonyl)(methyl)amino]thieno[3,2-d]pyrimidin-2-ylpiperazino)acetate (10): To a solution of $\mathbf{8}(2.5 \mathrm{~g}, 7.1 \mathrm{mmol})$ in DMF $(25 \mathrm{~mL})$ was added
TEA (1.44 g, $14.32 \mathrm{mmol})$ followed by ethyl bromo acetate (9) $(1.31 \mathrm{~g}, 7.8 \mathrm{mmol})$ at $0{ }^{\circ} \mathrm{C}$ and stirred the reaction at room temperature for $16 \mathrm{~h}$. After completion, reaction mixture was poured into ice cold water, extracted with ethyl acetate $(25$ $\mathrm{mL} \times 3$ ), combined extracts were washed with water, brine solution, dried the organic phase over anhy. $\mathrm{Na}_{2} \mathrm{SO}_{4}$ and evaporated the solvent to afford the cude product. The crude product was purified by column chromatography (elutent: $80 \%$ EtOAc: pet ether) to afford pure compound $\mathbf{1 0}$ as off white solid. Yield: $89 \%$; m.p.: $245-249{ }^{\circ} \mathrm{C}$; ${ }^{1} \mathrm{H} \mathrm{NMR}\left(400 \mathrm{MHz}, \mathrm{CDCl}_{3}\right)$ : $\delta 7.73-$ $7.71(\mathrm{~d}, J=5.5 \mathrm{~Hz}, 1 \mathrm{H}, \mathrm{ArH}), 7.13-7.12(\mathrm{~d}, J=5.5 \mathrm{~Hz} 1 \mathrm{H}$, ArH), 4.22-4.20 (q, $J=7.5 \mathrm{~Hz} 2 \mathrm{H}, \mathrm{CH}_{2}$-ester), 3.92 (brs, 4H, $\mathrm{CH}_{2}$ - piperazine), 3.39 (s, $3 \mathrm{H}, \mathrm{CH}_{3}$ ), 3.27 (s, $2 \mathrm{H}, \mathrm{NCH}_{2} \mathrm{CO}$ ), 2.67 (s, 4H, $\mathrm{CH}_{2}$-piperazine), 1.52 (s, 9H, $\left.\mathrm{C}\left(\mathrm{CH}_{3}\right)_{3}\right)$ 1.30-1.26 (q, $J=8 \mathrm{~Hz}, 2 \mathrm{H}, \mathrm{OCH}_{2}$-ester); ESI-MS: $m / z, 436.4(\mathrm{M}+\mathrm{H})^{+}$.

Synthesis of 2-(4-4-[(tert-butoxycarbonyl)(methyl)amino]thieno[3,2-d]pyrimidin-2-ylpiperazino)acetic acid (11): To a solution of compound $\mathbf{1 0}(2.5 \mathrm{~g}, 5.74 \mathrm{mmol})$ in methanol $(25 \mathrm{~mL})$ at $0{ }^{\circ} \mathrm{C}$ was added $6 \mathrm{~N} \mathrm{NaOH}(12 \mathrm{~mL})$ and stirred the reaction at room temperature for $8 \mathrm{~h}$. After completion, reaction mixture was poured into ice cold water, neutralized with aq. citric acid solution up to $\mathrm{pH} 5$ and filtered the formed precipitate to afford $\mathbf{1 1}$ as off white solid (Scheme-I). Yield: $93 \%$; m.p.: 128-129 ${ }^{\circ} \mathrm{C}$; IR (KBr, $\left.v_{\max }, \mathrm{cm}^{-1}\right)$ : 3435, 3004, 2979, 2940, 2869, 1698, 1638,1564, 1374, 1284, 1138, 983, 910, 794, 772; ${ }^{1} \mathrm{H}$ NMR (400 MHz, $\mathrm{CDCl}_{3}$ ): $\delta 8.18-8.17$ (d, $J$ $=5.2 \mathrm{~Hz}, 1 \mathrm{H}, \mathrm{ArH}), 7.19-7.17(\mathrm{~d}, J=5.2,1 \mathrm{H}, \mathrm{ArH}), 3.78$ (brs, $4 \mathrm{H}, \mathrm{CH}_{2}$-piperazine), 3.30 (s, $\left.3 \mathrm{H}, \mathrm{CH}_{3}\right), 3.20$ (s, $2 \mathrm{H}, \mathrm{NCH}_{2} \mathrm{CO}$ ), 2.64 (s, 4H, $\mathrm{CH}_{2}$-piperazine), $1.46\left(\mathrm{~s}, 9 \mathrm{H}, \mathrm{C}\left(\mathrm{CH}_{3}\right)_{3}\right)$; ESI-MS: $\mathrm{m} / \mathrm{z}, 408.3(\mathrm{M}+\mathrm{H})^{+}$.

General experimental procedure for synthesis of novel thienopyrimidine amide derivatives (13a-m): To a stirred solution of compound 11 (100 mg, $0.245 \mathrm{mmol}$ ) in DMF (4 $\mathrm{mL}$ ) was added diisopropylethylamine (DiPEA) (95 mg, 0.735 mmol), 2-(7-aza-1H-benzotriazole-1-yl)-1,1,3,3-tetramethyluronium hexafluorophosphate (HATU) (112 mg, $0.294 \mathrm{mmol})$ followed by addition of aryl or aliphatic amines (12a-m) (0.279 mmol) and stirred at room temperature for $18 \mathrm{~h}$. After completion reaction mixture was poured into ice cold water extracted with ethyl acetate $(2 \times 15 \mathrm{~mL})$, combined extracts were washed with brine solution, dried over anhy. $\mathrm{Na}_{2} \mathrm{SO}_{4}$ and concentrated under reduced pressure to afford crude product. This crude compound was purified by column chromatography (elutent: $2 \%$ methaol: $\mathrm{CH}_{2} \mathrm{Cl}_{2}$ ) to affords pure compounds $\mathbf{1 3 a}-\mathbf{m}$ as yields of the products varied between 80-94\%. By adapting this procedure the compounds $\mathbf{1 3 a - 1 3 m}$ were synthesized (Scheme-II).

tert-Butyl-2-(4-((cyclohexylcarbamoyl)methyl)piperazin1-yl)thieno[3,2-d]pyrimidin-4-ylmethylcarbamate (13a): Yield: $81 \%$; m.p.: $148-152{ }^{\circ} \mathrm{C}$; IR (KBr, $\left.v_{\max }, \mathrm{cm}^{-1}\right)$ : 3536 , 2972, 2855, 1712,1642, 1448, 1333, 1294, 1135, 1094, 1004, 930, 854, 578; ${ }^{1} \mathrm{H}$ NMR (400 MHz, $\mathrm{CDCl}_{3}$ ): $\delta$ 7.74-7.71 (d, $J=12,1 \mathrm{H}, \mathrm{ArH}), 7.14-7.13$ (d, $J=4 \mathrm{~Hz}, 1 \mathrm{H}, \mathrm{ArH}), 3.84$ (brs, 4H, $\mathrm{CH}_{2}$-piperazine), 3.80 (brs, 1H, CH-Cy.Hexyl), 3.39 (s, $3 \mathrm{H}, \mathrm{CH}_{3}$ ), 3.04 (s, 2H, $\mathrm{NCH}_{2} \mathrm{CO}$ ), 2.61 (brs, 4H, $\mathrm{CH}_{2}-$ piperazine), 1.92-1.89 (m, 2H. $\mathrm{CH}_{2}$-Cy.Hexyl), 1.68-1.58 (m, $2 \mathrm{H}, \mathrm{CH}_{2}$-Cy.Hexyl), 1.52 (s, 9H, C( $\left.\left.\mathrm{CH}_{3}\right)_{3}\right), 1.50-1.42$ (m, 2H, $\mathrm{CH}_{2}$-Cy.Hexyl); $1.27-1.20$ (m, 4H, $\left.\mathrm{CH}_{2}-\mathrm{Cy} \cdot \mathrm{Hexyl}\right) ;{ }^{13} \mathrm{C} \mathrm{NMR}$ 
<smiles>COC(=O)c1sccc1N</smiles><smiles>CCOC(=O)CN1CCN(c2nc(N(C)C(=O)OC(C)(C)C)c3sccc3n2)CC1</smiles>

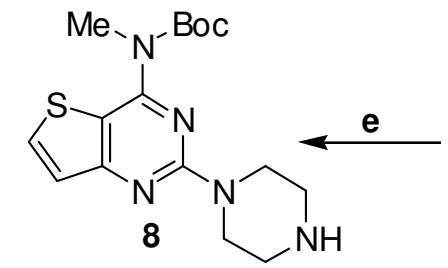<smiles>CC(=O)N(c1nc(Cl)nc2cc(C3CNCCN3)sc12)C(C)(C)C</smiles>

Reagents and conditions: a i) $\mathrm{KOCN}, \mathrm{AcOH}, \mathrm{H}_{2} \mathrm{O}$, room temperature, $16 \mathrm{~h}$; ii) $\mathrm{NaOH}$, room temperature, 4 h; b Toluene, NMP, $\mathrm{POCl}_{3}$, reflux, $16 \mathrm{~h}$; c THF, room temperature, $3 \mathrm{~h}$; d $(\mathrm{Boc})_{2} \mathrm{O}, \mathrm{DMF}$, TEA, room temperature, $4 \mathrm{~h}$; e DMF, $\mathrm{K}_{2} \mathrm{CO}_{3}$, room temperature, $12 \mathrm{~h}$; f DMF, TEA, room temperature, $16 \mathrm{~h}$; g MeOH, $6 \mathrm{~N} \mathrm{NaOH}$, room temperature, $8 \mathrm{~h}$

\section{Scheme-I}

(400 MHz, $\left.\mathrm{CDCl}_{3}\right): \delta 22.7,27.9,29.6,33.7,36.3,42.3,43.6$, $48.5,54.42,62.8,81.4,115.9,123.6,134.2,154.2,158.2$, 160.2, 163.7, 168.6; ESI-MS: $m / z, 489.4(\mathrm{M}+\mathrm{H})^{+}$.

tert-Butyl-2-(4-((cycloheptylcarbamoyl)methyl)piperazin-1-yl)thieno[3,2-d]pyrimidin-4-ylmethylcarbamate (13b): Yield: $85 \%$; m.p.: 174- $178{ }^{\circ} \mathrm{C}$; IR (KBr, $v_{\max }$, $\left.\mathrm{cm}^{-1}\right)$ : 3555, 2928, 1714, 1673, 1572, 1504, 1371, 1266, 1146, 1003; ${ }^{1} \mathrm{H}$ NMR (400 MHz, $\mathrm{CDCl}_{3}$ ): $\delta 7.75-7.73(\mathrm{~d}, J=8 \mathrm{~Hz}$, $1 \mathrm{H}, \mathrm{ArH}), 7.20-7.18$ (d, $J=8 \mathrm{~Hz}, 1 \mathrm{H}, \mathrm{ArH}), 4.03$ (brs, $1 \mathrm{H}$, CH-Cy.Heptyl), 3.87 (brs, 4H, $\mathrm{CH}_{2}$-piperazine), 3.39 (s, 3H, $\mathrm{CH}_{3}$ ), 3.03 (s, $2 \mathrm{H}, \mathrm{NCH}_{2} \mathrm{CO}$ ), 2.60 (brs, $4 \mathrm{H}, \mathrm{CH}_{2}$-piperazine), 1.92-1.90 (m, 2H, $\mathrm{CH}_{2}-\mathrm{Cy}$. heptyl), $1.70-1.60$ (m, 10H, $\mathrm{CH}_{2}-$ Cy. Heptyl), 1.53 (s, 9H, C( $\left.\left(\mathrm{CH}_{3}\right)_{3}\right) ;{ }^{13} \mathrm{C} \mathrm{NMR}(400 \mathrm{MHz}$, $\left.\mathrm{CDCl}_{3}\right): \delta 23.9,28.1,29.7,34.9,35.0,44.4,49.4,44.4,53.3$, $61.7,82.3,114.9,122.7,135.3,153.3,157.4,160.0,164.6$, 168.5; ESI-MS: $m / z, 503.1(\mathrm{M}+\mathrm{H})^{+}$.

tert-Butyl-N-methyl-N-[2-(4-2-[(2-morpholinoethyl)amino]-2-oxoethylpiperazino)thieno[3,2-d]pyrimidin-4yl]carbamate (13c): Yield: $92 \%$; m.p.: $106-110{ }^{\circ} \mathrm{C}$; IR (KBr, $\left.\mathrm{V}_{\max }, \mathrm{cm}^{-1}\right): 3526,3469,3320,3091,2930,2795,2840,2761$, $1710,1651,1564,1525,1450,1372,1334,1269,1151,1116$, 850,1002, 928, 861, 796, 762, 662, 584; ${ }^{1} \mathrm{H}$ NMR (400 MHz, $\left.\mathrm{CDCl}_{3}\right): \delta 7.75-7.7(\mathrm{~d}, J=8 \mathrm{~Hz}, 1 \mathrm{H}, \mathrm{ArH}), 7.14-7.13(\mathrm{~d}, J=4$, 1H, ArH), 3.89 (brs, 4H, $\mathrm{OCH}_{2}-$ Morpholine,), 3.71 (s, 4H, $\mathrm{CH}_{2}$-piperazine), 3.44-3.39 (m, 2H, $\mathrm{CH}_{2} \mathrm{NHCO}$ ); 3.39 (s, 3H, $\mathrm{CH}_{3}$ ), 3.07 (s, $2 \mathrm{H}, \mathrm{NCH}_{2} \mathrm{CO}$ ), 2.62 (brs, 4H, $\mathrm{CH}_{2}$-piperazine), 2.54-2.49 (m, 6H, $\mathrm{NCH}_{2}$-Morpholine \& $\left.\mathrm{NCH}_{2} \mathrm{C}\right), 1.52$ (s, 9H, $\left.\mathrm{C}\left(\mathrm{CH}_{3}\right)_{3}\right) ;{ }^{13} \mathrm{C} \mathrm{NMR}\left(400 \mathrm{MHz}, \mathrm{CDCl}_{3}\right): \delta 28.1,34.9,35.1$, 44.5, 53.3, 53.4, 57.1, 61.6, 67.1, 82.4, 114.9, 122.7, 135.3, 153.35, 157.4, 159.9, 164.6, 169.9; ESI-MS: $\mathrm{m} / \mathrm{z}, 520.4$ $(\mathrm{M}+\mathrm{H})^{+}$.
tert-Butyl-N-methyl-N-[2-(4-2-[(4-nitrobenzyl)amino]2-oxoethylpiperazino)thieno[3,2-d]pyrimidin-4-yl]carbamate (13d): Yield: $80 \%$; m.p.: $94-97^{\circ} \mathrm{C}$; IR $\left(\mathrm{KBr}, v_{\max }, \mathrm{cm}^{-1}\right)$ : 3528, 3466, 3320, 3080, 2976, 2931, 2842, 1711, 1649, 1550, $1525,1453,1370,1335,1261,1150,1001,1002,850,928$, $854,797,765,701,581 ;{ }^{1} \mathrm{H}$ NMR $\left(400 \mathrm{MHz}, \mathrm{CDCl}_{3}\right): \delta 8.22-$ 8.20 (d, $J=8 \mathrm{~Hz}, 2 \mathrm{H}, \mathrm{Ar}-\mathrm{H}), 7.75-7.71$ (d, $J=4 \mathrm{~Hz}, 1 \mathrm{H}, \mathrm{ArH})$, 7.47-7.45 (d, $J=8 \mathrm{~Hz}, 2 \mathrm{H}, \mathrm{Ar}-\mathrm{H}), 7.13-7.12$ (d, $J=4 \mathrm{~Hz}, 1 \mathrm{H}$, ArH), 4.62-4.61 (d, J = 4 Hz, 2H, NCH2Ar), 3.86 (brs, 4H, $\mathrm{CH}_{2}$-piperazine), 3.40 (s, $\left.3 \mathrm{H}, \mathrm{CH}_{3}\right), 3.16$ (s, $2 \mathrm{H}, \mathrm{NCH}_{2} \mathrm{CO}$ ), 2.64 (brs, 4H, $\mathrm{CH}_{2}$-piperazine), 1.52 (s, 9H, $\left.\mathrm{C}\left(\mathrm{CH}_{3}\right)_{3}\right) ;{ }^{13} \mathrm{C}$ NMR (400 MHz, $\mathrm{CDCl}_{3}$ ): $\delta 28.1,34.9,42.2,44.2,46.2,53.0$, 61.3, 67.0, 82.2, 114.8, 122.7, 135.2, 153.3, 157.4, 160.0, 164.6, 168.0; ESI-MS: $\mathrm{m} / z, 542.40(\mathrm{M}+\mathrm{H})^{+}$.

tert-Butyl-2-(4-((benzylcarbamoyl)methyl)piperazin1-yl)thieno[3,2-d]pyrimidin-4-ylmethylcarbamate (13e): Yield: $87 \%$; m.p.: $146-150{ }^{\circ} \mathrm{C}$; IR $\left(\mathrm{KBr}, \mathrm{v}_{\max } \mathrm{cm}^{-1}\right): 3531,3468$, 3101, 3080, 2971, 2838, 1713, 1665, 1556, 1520, 1371, 1328, 1266, 1152, 1091, 1001, 929, 799, 766, 622, 583; 1H NMR (400 MHz, $\left.\mathrm{CDCl}_{3}\right): \delta 7.76-7.74(\mathrm{~d}, J=6 \mathrm{~Hz}, 1 \mathrm{H}, \mathrm{ArH}), 7.53$ (brs, $1 \mathrm{H}, \mathrm{NH}), 7.37-7.26(\mathrm{~m}, 5 \mathrm{H}), 7.15-7.12(\mathrm{~d}, J=6 \mathrm{~Hz}, 1 \mathrm{H}$, ArH), 4.52-4.51 (d, J=4 Hz, 2H, $\mathrm{NCH}_{2} \mathrm{Ar}-\mathrm{H}$ ), 3.88-3.82 (brs, $4 \mathrm{H}, \mathrm{CH}_{2}$-piperazine), 3.38 (s, $3 \mathrm{H}, \mathrm{CH}_{3}$ ), 3.13 (s, 2H, $\mathrm{NCH}_{2} \mathrm{CO}$ ), 2.63 (brs, $4 \mathrm{H}, \mathrm{CH}_{2}$-piperazine), $1.52\left(\mathrm{~s}, 9 \mathrm{H},\left(\mathrm{CH}_{3}\right)_{3}\right) ;{ }^{13} \mathrm{C} \mathrm{NMR}$ $\left(400 \mathrm{MHz}, \mathrm{CDCl}_{3}\right): \delta 28.1,34.9,44.6,53.5,62.4,82.4,115.1$, $118.3,119.7,122.6,124.9,125.8,125.9,126.0,126.3,128.9$, $132.1,134.0,135.5,153.3,157.5,159.8,164.5,168.2$; ESIMS: $m / z, 497.42(\mathrm{M}+\mathrm{H})^{+}$.

tert-Butyl-2-(4-(((pyridin-2-yl)methylcarbamoyl)methyl)piperazin-1-yl)thieno[3,2-d]pyrimidin-4-ylmethylcarbamate (13f): Yield: $83 \%$; m.p.: $142-146{ }^{\circ} \mathrm{C}$; IR (KBr, 


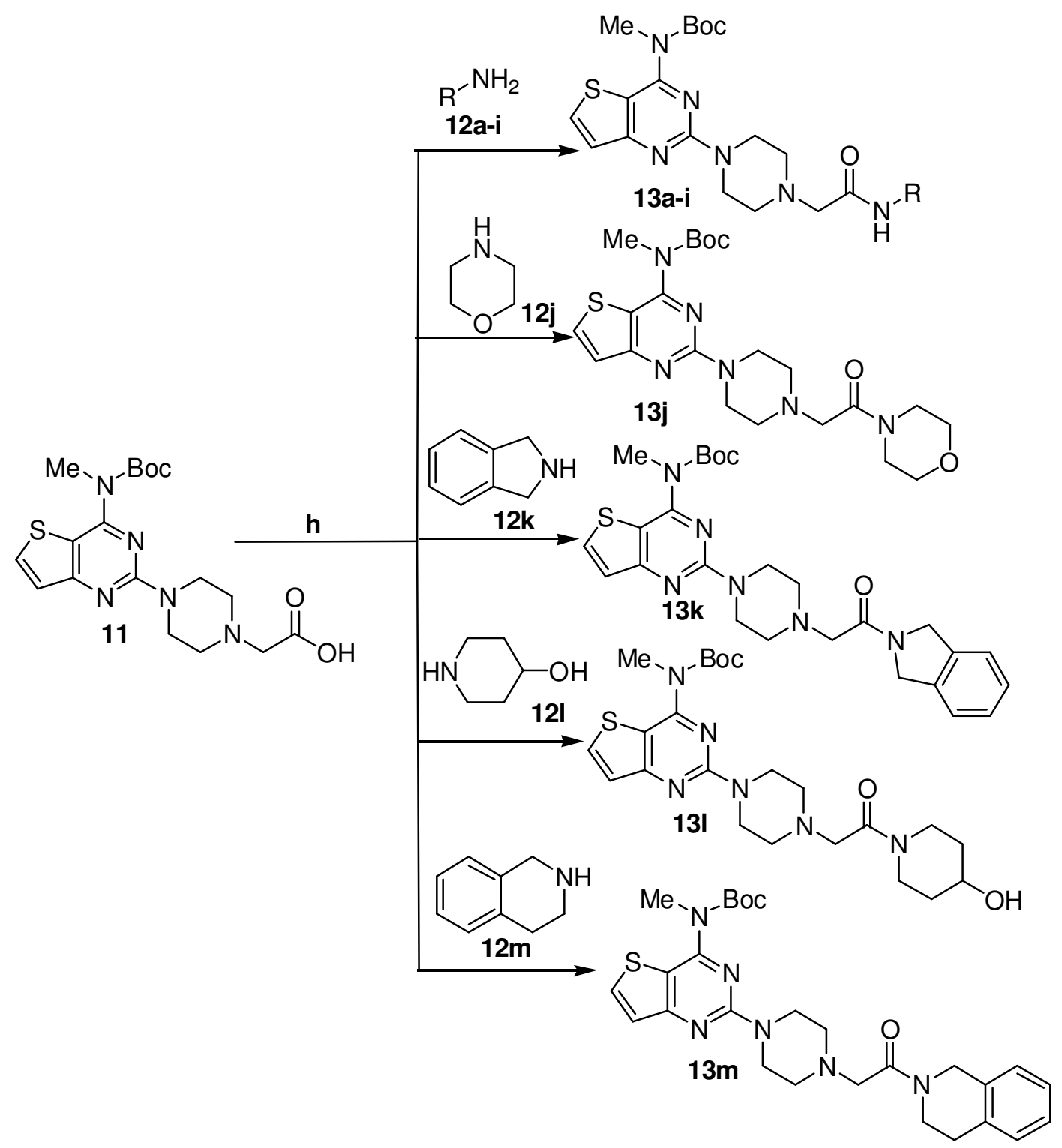

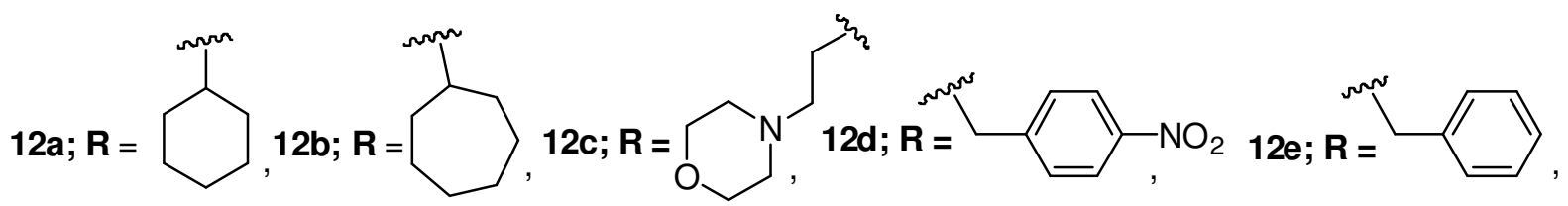<smiles>CCCCCCCCCCc1ccnn1C</smiles>

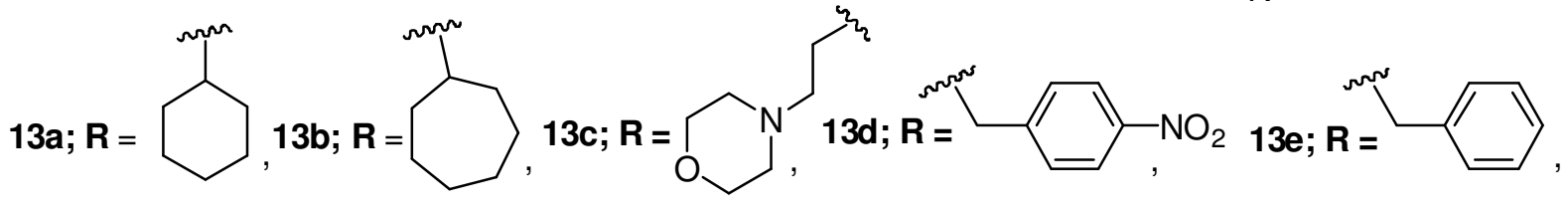

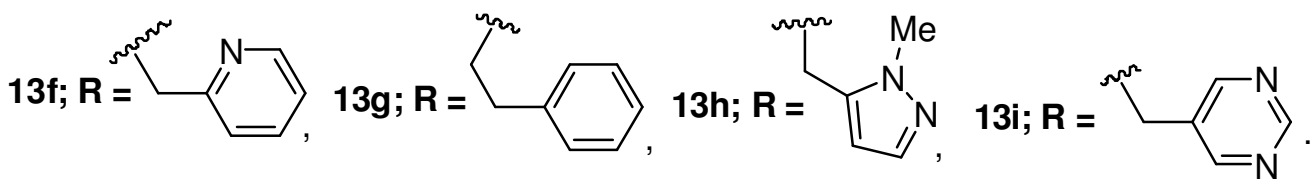

Reagents and conditions: h DMF, DIPEA, HATU, room temperature, $18 \mathrm{~h}$ 
$\left.v_{\max }, \mathrm{cm}^{-1}\right): 3343,3101,3043,2967,2924,2855,2837,1712$, $1669,1564,1519.9,1447,1373,1332,1303,1269,1150,1001$, 930, 798, 7667, 584; ${ }^{1} \mathrm{H}$ NMR $\left(500 \mathrm{MHz}, \mathrm{CDCl}_{3}\right): \delta 8.56-$ 8.55 (d, $J=5 \mathrm{~Hz}, 1 \mathrm{H}, \mathrm{Py}-\mathrm{H}), 8.24$ (brs, $1 \mathrm{H}, \mathrm{NH}), 7.74-7.73$ $(\mathrm{d}, J=5.5 \mathrm{~Hz}, 1 \mathrm{H}, \mathrm{ArH}) 7.68-7.71(\mathrm{t}, J=1.5 \mathrm{~Hz}, 1 \mathrm{H}, \mathrm{Py}-\mathrm{H})$, 7.29-7.27 (d, $J=8 \mathrm{~Hz}, 1 \mathrm{H}, \mathrm{Py}-\mathrm{H}), 7.21-7.20(\mathrm{~d}, J=5.5 \mathrm{~Hz}$, 1H, ArH), 7.14-7.13 (d, J = 5.5 Hz, 1H, Py-H), 4.63-4.62 (d, $J$ $=5 \mathrm{~Hz}, 2 \mathrm{H}, \mathrm{NCH} 2 \mathrm{Ar}$ ), 3.90 (brs, $4 \mathrm{H}, \mathrm{CH}_{2}$-piperazine), 3.39 (s, 3H, $\mathrm{CH}_{3}$ ), 3.14 (s, $2 \mathrm{H}, \mathrm{NCH}_{2} \mathrm{CO}$ ), 2.64 (brs, 4H, $\mathrm{CH}_{2-}$ piperazine $\left.), 1.52\left(\mathrm{~s}, 9 \mathrm{H},\left(\mathrm{CH}_{3}\right)\right)_{3}\right) ;{ }^{13} \mathrm{C} \mathrm{NMR}\left(400 \mathrm{MHz}, \mathrm{CDCl}_{3}\right)$ : $\delta$ 28.1, 29.6, 34.9, 44.1, 44.3, 44.6, 53.4, 61.7, 82.4, 114.8, $121.9,122.3,122.7,135.2,136.7,149.2,153.3,156.8,157.4$, 160.0, 164.6, 170.2; ESI-MS: $m / 2,498.4(\mathrm{M}+\mathrm{H})^{+}$.

tert-Butyl-2-(4-((phenethylcarbamoyl)methyl)piperazin1-yl)thieno[3,2-d]pyrimidin-4-ylmethylcarbamate (13g): Yield: $80 \%$; m.p.: $77-81{ }^{\circ} \mathrm{C}$; IR $\left(\mathrm{KBr}, \mathrm{v}_{\max }, \mathrm{cm}^{-1}\right)$ :3530. 3469, 3326, 3082, 2975, 2842, 2763, 1710, 1649, 1566, 1453, 1372, $1332,1263,1151,1002,928,854,796,758,701,581 ;{ }^{1} \mathrm{H}$ NMR (400 $\mathrm{MHz} \mathrm{CDCl}_{3}$ ): 7.74-7.73 (d, $\left.J=5.6 \mathrm{~Hz}, 1 \mathrm{H}, \mathrm{ArH}\right), 7.32-$ $7.21(\mathrm{~m}, 6 \mathrm{H}, \mathrm{Ar}-\mathrm{H}), 7.14-7.12(\mathrm{~d}, J=5.6 \mathrm{~Hz}, 1 \mathrm{H}, \mathrm{ArH}), 3.73$ (brs, 4H, $\mathrm{CH}_{2}$-piperazine), 3.62-3.60 (q, $J=2.1 \mathrm{~Hz}, 2 \mathrm{H}$, $\mathrm{NHCH}_{2} \mathrm{C}$ ), 3.39 (s, $3 \mathrm{H}, \mathrm{CH}_{3}$ ), 3.0 (s, $2 \mathrm{H}, \mathrm{NCH}_{2} \mathrm{CO}$ ), 2.89-2.85 $\left(\mathrm{t}, J=6.8 \mathrm{~Hz}, 2 \mathrm{H}, \mathrm{CCH}_{2} \mathrm{Ar}-\mathrm{H}\right), 2.49-2.47\left(\mathrm{~s}, 4 \mathrm{H}, \mathrm{CH}_{2}-\right.$ piperazine), $1.53\left(\mathrm{~s}, 9 \mathrm{H},\left(\mathrm{CH}_{3}\right)_{3}\right) ;{ }^{13} \mathrm{C} \mathrm{NMR}\left(400 \mathrm{MHz}, \mathrm{CDCl}_{3}\right)$ : $\delta 28.1,29.6,34.8,35.5,39.6,44.2,53.3,61.6,82.3,114.8$, 122.6, 126.6, 128.6,128.7, 135.3,1387, 153.3, 157.4, 159.8, 164.6, 169.9; ESI(M+H) ${ }^{+}$. MS: $m / z, 511.44(\mathrm{M}+\mathrm{H})^{+}$.

tert-Butyl-2-(4-(((1-methyl-1H-pyrazol-4-yl)methylcarbamoyl)methyl)piperazin-1-yl)thieno[3,2-d]pyrimidin4-ylmethylcarbamate (13h): Yield: $86 \%$; m.p.: 168-172 ${ }^{\circ} \mathrm{C}$; IR $\left(\mathrm{KBr}, \mathrm{v}_{\max }, \mathrm{cm}^{-1}\right): 3418,3392,3331,2980,2931,2850$, 2821, 1710, 1568, 1525, 1373, 1335, 1266, 1152, 1000, 931, 797, 763, 663, 582; ${ }^{1} \mathrm{H}$ NMR (400 MHz, $\mathrm{CDCl}_{3}$ ): $\delta$ 7.74-7.73 (d, $J=6 \mathrm{~Hz}, 1 \mathrm{H}, \mathrm{ArH}), 7.43(\mathrm{~s}, 1 \mathrm{H}, \mathrm{Ar}-\mathrm{H}), 7.36(\mathrm{~s}, 1 \mathrm{H}, \mathrm{CONH})$, 7.13-7.12 (d, $J=6 \mathrm{~Hz} 2 \mathrm{H}, \mathrm{ArH}), 4.36-4.34$ (d, $J=6 \mathrm{~Hz}, 2 \mathrm{H}$ ), $3.88\left(\mathrm{~s}, 3 \mathrm{H}, \mathrm{NCH}_{3}\right), 3.84$ (brs, $4 \mathrm{H}, \mathrm{CH}_{2}$-piperazine), 3.38 (s, $3 \mathrm{H}, \mathrm{CH}_{3}$ ), 3.07 (s, $2 \mathrm{H}, \mathrm{NCH}_{2} \mathrm{CO}$ ), 2.59 (s, $4 \mathrm{H}, \mathrm{CH}_{2}$-piperazine), $1.52\left(\mathrm{~s}, 9 \mathrm{H},\left(\mathrm{CH}_{3}\right)_{3}\right)$. ESI-MS: $\mathrm{m} / z, 501.1(\mathrm{M}+\mathrm{H})^{+}$.

tert-Butyl-2-(4-(((pyrimidin-5-yl)methylcarbamoyl)methyl)piperazin-1-yl)thieno[3,2-d]pyrimidin-4-ylmethylcarbamate (13i): Yield: $84 \%$; m.p.: $128-129{ }^{\circ} \mathrm{C}$; IR (KBr, $\left.V_{\max }, \mathrm{cm}^{-1}\right): 3452,3007,2983,1708,1659,1566,1386,1258$, 1147, 1003, 850, 728, 634; ${ }^{1} \mathrm{H}$ NMR (400 MHz, $\left.\mathrm{CDCl}_{3}\right): \delta$ 9.16 (s, 1H, Ar-H), 8.73 (s, 1H, Ar-H), 7.75-7.74 (m, 2H, ArH\&Ar-H), 7.14-7.13 (d, $J=6 \mathrm{~Hz}, 1 \mathrm{H}, \mathrm{ArH}), 4.8$ (brs, $1 \mathrm{H}, \mathrm{CONH}$ ), 4.54-4.53 (d, $J=6.4 \mathrm{~Hz}, 2 \mathrm{H}$. Ar-HCH2N), 3.86 (s, 4H, $\mathrm{CH}_{2}$-piperazine), $3.38\left(\mathrm{~s}, 3 \mathrm{H}, \mathrm{CH}_{3}\right), 3.14(\mathrm{~s}, 2 \mathrm{H}$, $\left.\mathrm{NCH}_{2} \mathrm{CO}\right), 2.62$ (s, $4 \mathrm{H}, \mathrm{CH}_{2}$-piperazine), $1.52\left(\mathrm{~s}, 9 \mathrm{H},\left(\mathrm{CH}_{3}\right)_{3}\right)$; ESI-MS: $m / z, 499.4(\mathrm{M}+\mathrm{H})^{+}$

tert-Butyl-methyl-2-(4-(2-morpholino-2-oxoethyl)piperazin-1-yl)thieno[3,2-d]pyrimidin-4-ylcarbamate (13j): Yield: $94 \%$; m.p.: $135-139^{\circ} \mathrm{C}$; IR $\left(\mathrm{KBr}, \mathrm{v}_{\max }, \mathrm{cm}^{-1}\right)$ : 3434, 3085, 2978, 2919, 2849, 2795, 1710, 1641, 1571, 1523, 1461, $1372,1264,1155,1111,1001,968,932,850,798,583 ;{ }^{1} \mathrm{HNMR}$ (400 MHz, $\mathrm{CDCl}_{3}$ ): $\delta$ 7.74-7.72 (d, $\left.J=8 \mathrm{~Hz}, 1 \mathrm{H}, \mathrm{ArH}\right), 7.15-$ 7.12 (d, $J=8 \mathrm{~Hz}, 1 \mathrm{H}, \mathrm{ArH}), 3.86$ (brs, 4H, OCH 2 -Morpholine), 3.74-3.64 (s, $4 \mathrm{H}, \mathrm{CH}_{2}$-piperazine), 3.40 (m, 7H, $\mathrm{NCH}_{2}$-Morpholine), 3.24 (s, $2 \mathrm{H}, \mathrm{NCH}_{2} \mathrm{CO}$ ), 2.61 (s, $4 \mathrm{H}, \mathrm{CH}_{2}$-piperazine), 1.52 (s, $\left.9 \mathrm{H},\left(\mathrm{CH}_{3}\right)_{3}\right) ;{ }^{13} \mathrm{C}$ NMR $\left(400 \mathrm{MHz}, \mathrm{CDCl}_{3}\right): \delta 28.3,34.7,45.4$, 54.4, 54.5, 57.54, 62.6, 67.5, 82.4, 115.1, 123.7, 135.5, 154.4, 156.4, 159.9, 165.5, 168.8; ESI-MS: $m / z, 477.1(\mathrm{M}+\mathrm{H})^{+}$.

tert-Butyl-2-(4-(2-(isoindolin-2-yl)-2-oxoethyl)piperazin-1-yl)thieno[3,2-d]pyrimidin-4-ylmethylcarbamate (13k): Yield: $93 \%$; m.p.: $91-95^{\circ} \mathrm{C}$; IR (KBr, $\left.v_{\max }, \mathrm{cm}^{-1}\right)$ : 3442 , 2977, 924, 2849, 1710, 1646, 1567, 1527, 1453, 1371, 1332, 1263, 1151, 1002, 930, 846, 796, 758; ${ }^{1} \mathrm{H}$ NMR (400 MHz, $\left.\mathrm{CDCl}_{3}\right): \delta 7.73-7.72(\mathrm{~d}, J=4 \mathrm{~Hz}, 1 \mathrm{H}, \mathrm{ArH}), 7.31-7.26(\mathrm{~m}, 4 \mathrm{H}$, Ar-H), 7.14-7.13 (d, J=4 Hz, 1H,ArH), 4.96 (s, 2H, $\mathrm{CONCH}_{2}$ ), 4.85 (s, 2H, $\mathrm{CONCH}_{2}$ ), 3.92 (brs, $4 \mathrm{H}, \mathrm{CH}_{2}$-piperazine), 3.39 (s, $3 \mathrm{H}, \mathrm{CH}_{3}$ ), 3.30 (s, 2H, $\mathrm{NCH}_{2} \mathrm{CO}$ ), 2.69 (s, 4H, $\mathrm{CH}_{2}$-piperazine), $1.54\left(\mathrm{~s}, 9 \mathrm{H},\left(\mathrm{CH}_{3}\right)_{3}\right) ;{ }^{13} \mathrm{C}$ NMR $\left(400 \mathrm{MHz}, \mathrm{CDCl}_{3}\right): \delta 28.1$, 34.9, 44.13, 44.27, 52.21, 52.37, 53.06, 53.40, 61.4, 82.3, $114.6,122.5,122.7,122.9,127.5,127.7,135.1,136.0,136.3$, 153.3, 157.3,160.0, 164.6, 168.3; ESI-MS: $m / z, 509.39$ $(\mathrm{M}+\mathrm{H})^{+}$.

tert-Butyl-2-(4-(2-(4-hydroxypiperidin-1-yl)-2-oxoethyl)piperazin-1-yl)thieno[3,2-d]pyrimidin-4-ylmethylcarbamate (13I): Yield: $93 \%$; m.p.: 119-123 ${ }^{\circ} \mathrm{C}$; IR (KBr, $\left.V_{\max }, \mathrm{cm}^{-1}\right): 545,3321,2928,2855,1714,1673,1572,1504$, 1333, 1266, 1147, 1094, 1050, 1003, 930, 854, 798, 708, 578; ${ }^{1} \mathrm{H}$ NMR (400 MHz, $\left.\mathrm{CDCl}_{3}\right): \delta 7.73-7.72(\mathrm{~d}, J=4 \mathrm{~Hz}, 1 \mathrm{H}$, ArH), 7.14-7.13 (d, $J=4 \mathrm{~Hz}, 1 \mathrm{H}, \mathrm{ArH}$ ), 4.10 (brs, 1H,CH$\mathrm{OH})$, 4.07-3.86 (m, 6H, $\mathrm{NCH}_{2} \mathrm{CO} \& \mathrm{CH}_{2}$-piperazine), 3.38 (s, $3 \mathrm{H}, \mathrm{CH}_{3}$ ), 3.2-3.18 (m, 4H, $\mathrm{CH}_{2}$-piperdine), 2.60-2.59 (s, 4H, $\mathrm{CH}_{2}$-piperazine) $1.90-1.85\left(\mathrm{~m}, 5 \mathrm{H}, \mathrm{CH}_{2}\right.$-piperdine \& $\left.\mathrm{OH}\right), 1.52$ $\left(\mathrm{s}, 9 \mathrm{H},\left(\mathrm{CH}_{3}\right)_{3}\right)$; ESI-MS: $\mathrm{m} / z, 491.39(\mathrm{M}+\mathrm{H})^{+}$.

tert-Butyl-2-(4-(2-(3,4-dihydroisoquinolin-2(1H)-yl)-2oxoethyl)piperazin-1-yl)thieno[3,2-d]pyrimidin-4ylmethylcarbamate (13m): Yield: $91 \%$; m.p.: 120-124 ${ }^{\circ} \mathrm{C}$; IR $\left(\mathrm{KBr}, v_{\max }, \mathrm{cm}^{-1}\right): 33440,3410,3095,2939,2832,1710$, 1634, 1564, 1373, 1261, 1154, 1001, 929, 849, 797, 763, 579; ${ }^{1} \mathrm{H}$ NMR (400 MHz, $\left.\mathrm{CDCl}_{3}\right): \delta 7.73-7.72(\mathrm{~d}, J=3 \mathrm{~Hz}, 1 \mathrm{H})$, 7.26-7.11 (m, 5H), 4.81-4.75 (m, 2H, $\left.\mathrm{CONCH}_{2}\right), 3.87-3.81(\mathrm{~m}$, $\left.6 \mathrm{H}, \mathrm{CH}_{2}\right), 3.37-3.32\left(\mathrm{~m}, 5 \mathrm{H}, \mathrm{NCH}_{3}, \mathrm{CH}_{2}\right), 2.96-2.88(\mathrm{~m}$, 2H, $\mathrm{CCH}_{2} \mathrm{Ar}-\mathrm{H}$ ), 2.61-2.60 (m, 4H, $\mathrm{CH}_{2}$-piperazine), 1.51 (s, $\left.9 \mathrm{H},\left(\mathrm{CH}_{3}\right)_{3}\right) ;{ }^{13} \mathrm{C} \mathrm{NMR}\left(400 \mathrm{MHz}, \mathrm{CDCl}_{3}\right): \delta 28.4,29.6,34.9$, 40.1, 43.3, 44.1, 44.2, 44.4, 47.2, 53.1, 61.6, 61.8, 82.2, 122.7, 126.4, 126.6, 134.0, 153.3, 157.4, 160.0, 164.6, 168.2, 168.4; ESI-MS: $m / z, 523.4(\mathrm{M}+\mathrm{H})^{+}$.

\section{RESULTS AND DISCUSSION}

The target compounds 13a-m was prepared as outlined in Schemes I and II. The compound methyl 3-aminothiophene2-carboxylate (1) was reacted with $\mathrm{KOCN}$ in acetic acid and $\mathrm{H}_{2} \mathrm{O}$ at room temperature for $16 \mathrm{~h}$. The resulting solid was filtered and treated with $50 \% \mathrm{NaOH}$ and reaction mixture was stirred at room temperature for $4 \mathrm{~h}$ to afford pure thieno[3,2d]pyrimidine-2,4-diol (2) in good yield. This diol intermediate (2) was reacted with $\mathrm{POCl}_{3}$ in toluene and NMP at reflux for $16 \mathrm{~h}$ to afford pure 2,4-dichlorothieno[3,2-d]pyrimidine (3) and intermediate 3 was coupled with $40 \%$ methylamine (4) in methanol and THF at room temperature for $3 \mathrm{~h}$ to afford $N$ (2-chlorothieno[3,2- $d$ ]pyrimidin-4-yl)- $N$-methylamine (5) in $91 \%$ yield. The intermediate 5 undergoes to protection reacting with (Boc) $)_{2} \mathrm{O}$ in DMF and triethylamine at room temperature 
for $4 \mathrm{~h}$ to afford protected compound $\mathbf{6}$, it was coupled with piperizine (7) in DMF, $\mathrm{K}_{2} \mathrm{CO}_{3}$ at room temperature for $12 \mathrm{~h}$ to afford pure tert-butyl $N$-methyl- $N$-(2-piperazinothieno[3,2$d$ ]pyrimidin-4-yl)carbamate (8) in $82 \%$ yield. The piperizine intermediate 8 was reacted with ethylbromoacetate (9) in DMF, triethylamine at room temperature for $16 \mathrm{~h}$ to afford pure compound ethyl 2-(4-4-[(tert-butoxycarbonyl)(methyl)amino]thieno[3,2- $d]$ pyrimidin-2-ylpiperazino)acetate (10). This ethylester intermediate $\mathbf{1 0}$ was undergoes to basic hydrolysis with $6 \mathrm{~N} \mathrm{NaOH}$ in methanol at room temperature for $8 \mathrm{~h}$ to afford pure acid intermediate $\mathbf{1 1}$ in $\mathbf{8 2} \%$ yield as shown in Scheme-I. The acid intermediate $\mathbf{1 1}$ was coupled with different substituted amines (12a-m) in DMF, DIPEA and HATU at room temperature for $18 \mathrm{~h}$ to afford pure amide compounds (13a-m) in excellent yield.

Biological assay: The synthesized thienopyrimidine amide derivatives 13a-m were dissolved in dimethyl sulphoxide at $30 \mu \mathrm{g} / \mu \mathrm{L}$ concentration (standard antibacterial drug, ampicilline was used as the reference antibiotic) and tested against Gramnegative strains of (1) Escherichia coli, (2) Klebsiella. pneumonia and Gram-positive strains of (3) Staphylococcus aureus and (4) Bacillus subtilis using agar well diffusion method according to the literature protocol $[12,13,27,28]$. Activity was determined by zones showing complete inhibition (mm). Growth inhibition was calculated with reference to positive control. All the samples were taken in triplicates.

Antibacterial activity: These newly synthesized thienopyrimidine derivatives (13a-m) were evaluated for their antibacterial activity against two Gram-negative and two Grampositive bacterial strains viz., Escherichia coli, Klebsiella pneumonia, Staphylococcus aureus and Bacillus subtilis. The results are summarized in Table- 1 and ampicilline was used as positive control.

TABLE-1

in vitro ANTIBACTERIAL ACTIVITY OF COMPOUNDS 13a-m (CONCENTRATION USED $30 \mu \mathrm{g} / 20 \mu \mathrm{L}$ OF DMSO)

\begin{tabular}{ccc|cc}
\hline \multirow{2}{*}{ Compd. } & \multicolumn{2}{c}{ Zones of inhibition of compounds 13a-m in mm } \\
\cline { 2 - 5 } & \multicolumn{2}{c}{ Gram-negative } & \multicolumn{2}{c}{ Gram-positive } \\
\cline { 2 - 5 } E. coli & K. pneumonia & S. aureus & B. subtilis \\
\hline 13a & 4 & 6 & 3 & 3 \\
13b & 0 & 3 & 4 & 0 \\
$\mathbf{1 3 c}$ & 0 & 5 & 4 & 0 \\
$\mathbf{1 3 d}$ & 4 & 3 & 5 & 6 \\
$\mathbf{1 3 f}$ & 0 & 0 & 5 & 5 \\
$\mathbf{1 3 g}$ & 5 & 7 & 2 & 0 \\
$\mathbf{1 3 h}$ & 3 & 4 & 0 & 0 \\
$\mathbf{1 3 i}$ & 8 & 5 & 4 & 4 \\
$\mathbf{1 3 j}$ & 0 & 5 & 2 & 7 \\
$\mathbf{1 3 k}$ & 12 & 13 & 17 & 15 \\
$\mathbf{1 3 m}$ & 5 & 6 & 0 & 6 \\
Ampicilline & 16 & 14 & 17 & 16 \\
\hline
\end{tabular}

It is observed that compounds $\mathbf{1 3 g}, \mathbf{1 3 i}, \mathbf{1 3 k}$ and $\mathbf{1 3 m}$ revealed excellent antibacterial activity with zone of inhibition 30-33 mm against $E$. coli (Gram-negative bacteria) and $S$. aureus (Gram-positive bacteria) even in the case of $P$. aeruginosa (Gramnegative bacteria) and $S$. pyogenes (Gram-positive bacteria), compounds 13d, 13k and $\mathbf{1 3 f}$ displayed excellent anti-bacterial activity with zone of inhibition 22-25 mm (Table-1).
REFERENCES

1. R. Klevens, J. Edwards, C. Richards Jr., T. Horan, R. Gaynes, D. Pollock and D. Cardo, Public Health Rep., 122, 160 (2007); https://doi.org/10.1177/003335490712200205.

2. R. Kharb, P.C. Sharma and M.S. Yar, J. Enzyme Inhib. Med. Chem., 26, 1 (2011); https://doi.org/10.3109/14756360903524304.

3. R. Kharb, M. Shahar Yar and P. C. Sharma, Curr. Med. Chem., 18, 3265 (2011);

https://doi.org/10.2174/092986711796391615.

4. R. Kharb, M. Shahar Yar and P.C. Sharma, Mini Rev. Med. Chem., 11, 84 (2011); https://doi.org/10.2174/138955711793564051.

5. V.S. Dinakaran, B. Bhargavi and K.K. Srinivasan, Pharmachem, 4, 255 (2012).

6. M. Lindvall, C. McBride, M. McKenna, T.G. Gesner, A. Yabannavar, K. Wong, S. Lin, A. Walter and C.M. Shafer, ACS Med. Chem. Lett., 2, 720 (2011);

https://doi.org/10.1021/ml200029w.

7. Y. Ni, A. Gopalsamy, D. Cole, Y. Hu, R. Denny, M. Ipek, J. Liu, J. Lee, J.P. Hall, M. Luong, J.-B. Telliez and L.-L. Lin, Bioorg. Med. Chem. Lett., 21, 5952 (2011); https://doi.org/10.1016/j.bmcl.2011.07.069.

8. T.P. Heffron, M. Berry, G. Castanedo, C. Chang, I. Chuckowree, J. Dotson, A. Folkes, J. Gunzner, J.D. Lesnick, C. Lewis, S. Mathieu, J. Nonomiya, A. Olivero, J. Pang, D. Peterson, L. Salphati, D. Sampath, S. Sideris, D.P. Sutherlin, V. Tsui, N.C. Wan, S. Wang, S. Wong and B. Zhu, Bioorg. Med. Chem. Lett., 20, 2408 (2010); https://doi.org/10.1016/j.bmcl.2010.03.046.

9. D.P. Sutherlin, D. Sampath, M. Berry, G. Castanedo, Z. Chang, I. Chuckowree, J. Dotson, A. Folkes, L. Friedman, R. Goldsmith, T. Heffron, L. Lee, J. Lesnick, C. Lewis, S. Mathieu, J. Nonomiya, A. Olivero, J. Pang, W.W. Prior, L. Salphati, S. Sideris, Q. Tian, V. Tsui, N.C. Wan, S. Wang, C. Wiesmann, S. Wong and B. Zhu, J. Med. Chem., 53, 1086 (2010); https://doi.org/10.1021/jm901284w.

10. A.B.A. El-gazzar, H.A.R. Hussein and H.N. Hafez, Acta Pharm., 57, 395 (2007); https://doi.org/10.2478/v10007-007-0032-6.

11. D.P. Sutherlin, L. Bao, M. Berry, G. Castanedo, I. Chuckowree, J. Dotson, A. Folks, L. Friedman, R. Goldsmith, J. Gunzner, T. Heffron, L. Lesnick, C. Lewis, S. Mathieu, J. Murray, J. Nonomiya, J. Pang, N. Pegg, W.W. Prior, L. Rouge, L. Salphati, D. Sampath, Q. Tian, N.C. Tsui, N.C. Wan, S. Wang, B.Q. Wei, C. Wiesmann, P. Wu, B.-Y. Zhu and A. Olivero, J. Med. Chem., 54, 7579 (2011); https://doi.org/10.1021/jm2009327.

12. J.J. Wallin, K.A. Edgar, J. Guan, M. Berry, W.W. Prior, L. Lee, J.D. Lesnick, C. Lewis, J. Nonomiya, J. Pang, L. Salphati, A.G. Olivero, D.P. Sutherlin, C. O’Brien, J.M. Spoerke, S. Patel, L. Lensun, R. Kassees, L. Ross, M.R. Lackner, D. Sampath, M. Belvin and L.S. Friedman, Mol. Cancer Ther, 10, 2426 (2011); https://doi.org/10.1158/1535-7163.MCT-11-0446.

13. S. Mathieu, S.N. Gradl, L. Ren, Z. Wen, I. Aliagas, J. Gunzner-Toste, W. Lee, R. Pulk, G. Zhao, B. Alicke, J.W. Boggs, A.J. Buckmelter, E.F. Choo, V. Dinkel, S.L. Gloor, S.E. Gould, J.D. Hansen, G. Hastings, G. Hatzivassiliou, E.R. Laird, D. Moreno, Y. Ran, W.C. Voegtli, S. Wenglowsky, J. Grina and J. Rudolph, J. Med. Chem., 55, 2869 (2012); https://doi.org/10.1021/jm300016v.

14. B.S. Safina, S. Baker, M. Baumgardner, P.M. Blaney, B.K. Chan, Y.-H. Chen, M.W. Cartwright, G. Castanedo, C. Chabot, A.J. Cheguillaume, P. Goldsmith, D.M. Goldstein, B. Goyal, T. Hancox, R.K. Handa, P.S. Iyer, J. Kaur, R. Kondru, J.R. Kenny, S.L. Krintel, J. Li, J. Lesnick, M.C. Lucas, C. Lewis, S. Mukadam, J. Murray, A.J. Nadin, J. Nonomiya, F. Padilla, W.S. Palmer, J. Pang, N. Pegg, S. Price, K. Reif, L. Salphati, P.A. Savy, E.M. Seward, S. Shuttleworth, S. Sohal, Z.K. Sweeney, S. Tay, P. Tivitmahaisoon, B. Waszkowycz, B. Wei, Q. Yue, C. Zhang and D.P. Sutherlin, J. Med. Chem., 55, 5887 (2012); https://doi.org/10.1021/jm3003747.

15. M.I. Crespo, L. Pages, A. Vega, V. Segarra, M. Lopez, T. Domenech, M. Miralpeix, J. Beleta, H. Ryder and J.M. Palacios, J. Med. Chem., 41, 4021 (1998); https://doi.org/10.1021/jm981012m. 
16. Y. Kotaiah, N. Harikrishna, K. Nagaraju and C. Venkata Rao, Eur. J. Med. Chem., 58, 340 (2012); https://doi.org/10.1016/j.ejmech.2012.10.007.

17. H.A. Stefani, C.B. Oliveira, R.B. Almeida, C.M.P. Pereira, R.C. Braga, R. Cella, V.C. Borges, L. Savegnago and C.W. Nogueira, Eur. J. Med. Chem., 41, 513 (2006); https://doi.org/10.1016/j.ejmech.2006.01.007.

18. H. Kikuchi, K. Yamamoto, S. Horoiwa, S. Hirai, R. Kasahara, N. Hariguchi, M. Matsumoto and Y. Oshima, J. Med. Chem., 49, 4698 (2006); https://doi.org/10.1021/jm0601809.

19. B. Singh, A. Maheshwari, G. Dak, K. Sharma and G.L. Talesara, Indian J. Pharm. Sci., 72, 607 (2010): https://doi.org/10.4103/0250-474X.78529.

20. S. Rostamizadeh, M. Nojavan, R. Aryan, H. Sadeghian and M. Davoodnejad, Chin. Chem. Lett., 24, 629 (2013); https://doi.org/10.1016/j.cclet.2013.04.035.

21. H.N. Hafez, H.A. Hussein and A.R. El-Gazzar, Eur. J. Med. Chem., 45, 4026 (2010);

https://doi.org/10.1016/j.ejmech.2010.05.060.
22. H.M. Aly, N.M. Saleh and H.A. Elhady, Eur. J. Med. Chem., 46, 4566 (2011); https://doi.org/10.1016/j.ejmech.2011.07.035.

23. B.V. Ashalatha, B. Narayana, K.K. Vijaya Raj and N.S. Kumari, Eur. J. Med. Chem., 42, 719 (2007); https://doi.org/10.1016/j.ejmech.2006.11.007.

24. T. George, C.L. Kaul, R.S. Grewal and R. Tahilramani, J. Med. Chem., 14, 913 (1971); https://doi.org/10.1021/jm00292a005.

25. O. Bruno, C. Brullo, A. Ranise, S. Schenone, F. Bondavalli, E. Barocelli, V. Ballabeni, M. Chiavarini, M. Tognolini and M. Impicciatore, Bioorg. Med. Chem. Lett., 11, 1397 (2001); https://doi.org/10.1016/S0960-894X(01)00221-9.

26. R.K. Russell, J.B. Press, R.A. Rampulla, J.J. McNally, R. Falotico, J.A. Keiser, D.A. Bright and A. Tobia, J. Med. Chem., 31, 1786 (1988); https://doi.org/10.1021/jm00117a019.

27. L.M. Lima, F.S. Frattani, J.L. Dos Santos, H.C. Castro, C.A.M. Fraga, R.B. Zingali and E.J. Barreiro, Eur. J. Med. Chem., 43, 348 (2008); https://doi.org/10.1016/j.ejmech.2007.03.032.

28. P.B. Nariya, N.R. Bhalodia, V.J. Shukla and M.B. Nariya, Int. J. Pharm. Tech. Res., 2, 2522 (2010). 